\title{
[ 320$]$
}

platt gedrückt werden. Diefe Federn liegen mehrfach auf einander, fo dafs diejenigen, die der Haut des Vogels zunächlt liegen, nur eine fehr vermin. derte Linwirkurgy des äulsern Luftltolses erleiden.

Ueberdies werden diele Federn voin Vogel be-

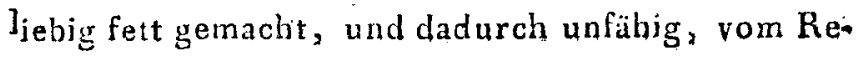
gen benetzt zu werden,

\section{NACHTRAG.}

x. Etzeas über die Flugmafohine des Herrn Degen in Wien.

Gefcliritben zu biünn an soten Qctober s808.

Ueber diefe Flugmafchine wirl fchon feit längerer Zeit vieles gefprochen, Oeffentliche in-und ausländifche Blätter erzählen davon, und Mancher freut fich wohl fchon im Stillen der gliicklichen Erfindung, die das theure Poftgeld zu eriparen, den lang erfehnten Kaiffee, zum Trotz der Länderfperre, über die Grïnze zu fübren, oder auch einen bedrängten Erdeufuhn in gläcklichere Gefilde zu bringen dienen wird, Wie mancher Uhrmacher hat nicht fchon gejubelt, dafs der zweite lkarus unter ihnen hat erftehen follen? unter andern folrieb ein franzöfifcher horlogier de fa Majefié l'Empereur mit künltlerifcher Selbftgenüglichkeit an Herrn $\mathrm{D}$ e gen, (einer meirer Freunde hat den Brief gelefen,) es freue ihn, endlich einen Genolfen feiner Kunft gefunden zu baben, der fich durch cie merkwiirdigfto 


\section{[ $32 \mathrm{r}$ ]}

Erfindung auf dem Erdboden einen unfterblichen Namen bereite, und, er könne darum nicht umbin, iln feiner Wohlgeneigtheit zu verfichern.

Es ift hier nur zu fehen, ob und was durch Herrn Degen's Verfuche mit feiner Flugmafchine für die Phylik gewonnen wird. Icb habe feit ihrer Entftebung manigfaltige Erkundigungen durch Augenzeugen von ibr eingezogen, atch felbft eine Zeichnurg von ihr vor mir liegen. Nian verfichert, Herr Degen habe feine Idee aus Unterfuchungen über die Flugwerkzeuge der Vügel gefchopft, und fich befonders aus dem Baue der Käfer viel wichtiges abftrahirt. Ich, meines Theils, habe mannigfaltige Unterfuchungen über das Fliegen der Vögel angeftellt, und durch fehr viele Zergliederungen, von der Fledermaus an bis zu den grölsten Falkenarten, mir die genauefte Kenntuifs der Flugwerkzeuge und des Flugrmechanismus der Vögel exworben. *) Aber ich kann verfichern, dals Herrn Degen's Flugmafchine mit den Flugwerkzeugen der Vögel, weder in ihrer Structur noch in ihren Functionen, die mindefte Aehnlichleit hat. Der Zeichnung und Befchreibung nach gleichen Herrn Degen's Flägel den Deckflügeln der Käfer: allein diefe Schalen dienen den Käfern nicht zum Fluge,

*) Ich werde Tpäterhin eine Darfiellung digfer Unierer. fuchungen' und meiner Theorie des Fliegens in ei ner unfrer phyfikalifchen Zeitfchriften bekannt machen, 


\section{[ 322 ]}

fondern find nur die Decken, welche die feinen und bygrofkopi[chen Flügel diefer Infekten vor Verletzung und Näfle fobutzen, und die bei ihrem Fluge unbeweglich ausgelpannt gehaiten werden. Die Flügel diefer Thiere find fehr fein und künftlich gebaut, und beftehen aus einer feinen Membran, die $z$ wifchen fehr dünnen mit Gelenken verlebenen Knochen ausgefpannt ift, wie die der Fledermäufe, Von allem dem zeigt Degen's Flugmafchine fo wenig, als von den Flügeln der Vögel. Seine Flägel find fehr mühfam mit fehr vielen Papierklappen iberlegt, die fich nach unten öfnen, und durch einen gemeinfchaftlichen $\mathrm{Zug}$ von eben fo vielen Fäden geöffnet und gefchiolfen werden, um dem Drucke der Luft, der diefes bei der Auf - und Niederbewegung der Flügel ohnehin bewirkt, zu Hülfe zu liommen.

Bei Herrn Degén's Flïgel findet daher im Tücikfchlage ein viel zu beträchtlicher Wiclerftand Statt, (f. die vorfteh. Abhandlung.) Die Flugwerkzeuge der Vögel und ihre Bewegungen find mit ei-

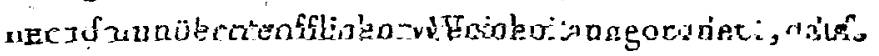
der menfchliche Verftand es fich zum Gläcke rechnen mufs, weun er fie zu erkennen vermag: etwas befferes oder gleich gut wirkendes an ihre Stelle zu fetzen, liegt weit jenfeits der Grenze feines Vermögens. Darum nun ift Hrn. Degen's Flugmafchine nicht einmahl eine Annäherung an jene Vorrichtung, die den Menfchen in die Region der Vögel $z u$ tragen rermöchte. 


\section{[ $\begin{array}{ll}323 & ]\end{array}$}

Herr $D$ egen erhebt fich vermittelit eines $G e$. gengewichts von 75 ffunden durch feine Flügel, oder, da er felbft mit der Mafchine 1 f́f Pfund wiegt, überwindet er durch den vermittelft des Nieder: fchlags der Flügel erzeugten Widerftand in der. Luft, (nach Abzug der Wirkung des Rück[chlares,) eine Laft von 69 , oder, mit Einfchlufs von 9 Pfund Reibung, von 78 Pfunden. Und hierin bat er fich nur allerdings ein Verdienlt erworben, dafs er den Menfchen vor Augen gezeigt hat, dafs es möglich ift, durch blofsen Widerftand in der federleichten Luft eine Laft von 69 Pfund $z u$ erbeben; ein Experiment, das wohl für manche Phyfiker fo wenig überflüffig mag gewefen feyn, als für jene Wiener, die da behaupteten, dais Degen's Fliegen keine Kunft fey, indem iha das Gegengewicht von 75 Plund in die Höhe ziehe.

Kennte ich die nöthigon Dimenfionen von Hrn. Degen's *) Flugwerk, fo würde es leicht feyn, nach den vorigen Grundfätzen und meinen Verfuchen über den abfoluten Widerftand der Luft, ( $A n$ nalen der $P h y / 2 k$, B. 23, S. 130,) die Laft zu beftimmen, die mit den Flügeln konnte gehoben werden; auch hätte man daraus das nöthige Gegengewicht a priori berechnen können, ohne dafs Herr Degen es durch mühfame Verfuche erlt zu finden

*) Man findet fie in Heft 9 diefer Annalen, S. 6, wor. nach ich auch die vorftehenden Zahlen bièr berichtigt habe.

Gilb. 


\section{[ 324 ]}

genöthigt gewolen wäre. Aus der Gröfse der Laft kann eben fo die Gröfse dér Flügel felbft, bei befitimmter Gefshwindigkeit ihrer Bewegung, fo wie diefe bei beftimmter Flügelgröfse, und lo weiter, durch Rechoung angegeben iver:le.1. Herrn Degen's Verfuche Gnd allo vicht gesignet, for die Phylik etwas Natzliches zu liefern. Durch meine Verfurhe, (a. a. O.,) habe ich den Wilpirftand in der Luft bis zu einer Genauigkeit heftinmt, welche zu den feinften Bereshnungen aller Art aber diefen Gegenftand ausreichend ift.

Die Grobse der Fluggel ift aberhanpt keine Hauptfunction des Werthes finr die fliegende Laft. Man fieht aus der im vorfiehenclen Auflatze angegebenen Formel, dals bai einerlei zu erbebender Laft die Flügel an Breite unci Länge grofs oder klein feyn künnen, wenn nur im letzten Falle der Schlagwinkel, vorzüglich aber die Zahl der Flügelfchläge in der Secunde, grof́s find. Es kümmt auf die vor: handenen Berlingungen, auf die Stārke cler Muskelkraft unil andere Momente ạn, zu beftimmen, welche ron diefen Gröfsen man zur Hauptfunction nehmen muls, wenn man durch Flug eine lebendo oder todte Laft fich will erheben laflen. Hierzu find mancherlei phyfiologifche and mecbanifche Unterfuchungen erforderlich. Aber Herrn Degen foheinen $z \mu$ denfelben die nöthigften mathematifchen Kenntniffe abzugehen, walche hierbei doch nothwendiger find, als beim Uhrmacher die Theilfoheibe, 


\section{[ $\left.\begin{array}{lll}325 & \end{array}\right]$}

Herr Degen hat den ungläcklichen Gedanken, feine l'lugmafchine zur Direction des Luftballous zu verwenilen; ein Gedanke, deffen Ausfïhrung, wean er am befien gelärge, ihm unfehlbar den Fials koften würde, und von welchem abzuftehen, ich ihn recht inftänlig bitte. Sein unfehlbarer Sturz, wenn es ihm gelingt, foch wirklich bei Windlitle zu erheben, ilt mathematifch zu erweifen. Herr Degen foheint keinen Begriff davon zu haben, welche mannigfaltig entgegenftrebende Kriffte bei einer folchen Operation da oben ins Spiel kommen würden. Die horizontale Bewe. gung des Ballons durch den Wind wïrde ihn aufser der fenkrechten Linie halten und die Wirkung feiner Flügel unnütz machen, da feiner Mafchine alle jene Hülfsmittel fehlen, durch welche der Vogel fich in der Horizontalebene erhält. Selblt ein Vogel, mit einer Laft verfehen, welche durch die Steigkraft eines über ihm befeftigten Ballons aufyehoben wärde, wäre nicht im Stande, fich auch nur. wenige Augenblicke in der Luft zu erhalten. Freilich kann fich Herr Degen feiner Flügel als eines Fallfchirms bedienen, und vermittelft jhrer in der kaiferlichen Reitfchule in Wien aus einer Höhe von 5o Fufs niederfinken: aber hier wird er durch den Strick, an welchem das Gegengewicht, (über der Saaldecke,) hängt, fonkrecht erhalten, welches beim freien Falle in der Luft keinen Augenblick Statt finden würde. Die Form des Fallfchirms ift einzig und allein auf die kreisrunde befchränkt. 


\section{$\left[\begin{array}{ll}326 & ]\end{array}\right.$}

Aus diefem allen folgt, dals Herrn Degen's Verfuche weder für die Theorie des Fliegens noch für die des Widerftandes in der Luft einigen Nutzen gewähren. Auch wird er es, bei allen Vervollkommnungen feiner Mafchire, nie dahin bringen, fich frei in der Luft zu erbeben; er mufste denn feine Ideen und Formen gänzlich verlaffen und die wahren aufnehmen. Herr Degen foll ein gefchickter Künftler feyn, und in diefer Rückficht könnte er bei feinem Eifer, wenn er richtiger Auleitung Folge leiften wollte, hierin viel nützen: ein jeder weifs, wie viel bei mechanifchen Ausfuhtrungen auf Luft und Gefchicklichkeit des Arbeiters ankömmt. Ich habe ihn eintnahl gelegentlich antragen laffen, ihm mit nähern Anleitungen zum Bau einer folchen Mafchine an die Hand zu gehen, weils aber nicht, ob es ihm ausgerichtet worden ift. Ueberhaupt weifs man, dafs Männer von folchen einmahl miit Eifer ergrifienen Ideen auch durch das Beffere nicht leicht abzubringen fond.

Hiermit fage ich nicht, dals es den Menfchen unmöglich fey, fich, den Vögeln gleich, in die Luft zu crheben. Im Gegentheile, ich glaube mit unumftölslichen Gründen beweifen zu können, dafs es dem Menfchen fehr wohl möglich ift, in der Luft zu fliegen: noch mebr, ich erbiete mich, durch Hülfe von Geld und einer gefchickten Arbeiter eine Mafchine zu bauen, in welcher ein Menfch mit allen möglichen Flugbewegungen die Lüfte fo leicht foll durchftreichen können, wie der leicht gefiederte Falke. Ift 


\section{$\left[\begin{array}{ll}327 & 1\end{array}\right.$}

ja doch der Menfch mit der Schnelligkeit des leicht fülsigen Pferdes über grofse Landftriche hinweggeeilet; hat er foch die ungeheuren Meere über ihre Bewohner hinweg durchlchwommen; warum foll nicht endlich auch die Zeit kommen, in der er gleich den Vügeln fich durch die Lüfte f́h wingt?

Nur wird durch Herrn Degen's Flugverfich zu diefer Kunft kein Schritt geihan. Aber hierin ift man ihm allen Dank fchulitig, dafs er durch handgreifliche Verfuche die Aufmerkfamkeit des Publicums wieder auf einen Gegenftand gelenkt hat, von dem man kaum mehr fprechen konnte, ohne fich lächerlich zu machen, (weil die wichtigen Unterfuchungen darüber nie gründlich waren angeftellt worden;) und dafs er dadurch vielleiche die Ausführung des erften Verfuches der Menlchen, sleich dern Vogel zu fliegen, früher herbei führt. So hat wobl auch Cavendifb Seifenblafen in die Höbe fteigen laflen; aber erft mehr als 20 Jahre wachher hat Charles einen Luftbalion gefült, und ift damit felbft in die Höhe geftiegen.

$$
\text { Prechtl. }
$$

2. Ueber Degen's neueften Flugverfuch in Verbindung mit einem Luftballon. Aus einem Briefe an den Prof. Gilbert.

Wien den 15ten Nor. r8os.

Ueber die Theorie des Vügelfluges kümmt jetzt von fo verfchiedenen Seiten her fo manches zur 